\title{
Inventaire des ptéridophytes dans le Parc National d'El Kala (Algérie orientale)
}

Hamel Tarek ${ }^{1}$, Boulemtafes Amir², Bellili Abdelmalek ${ }^{1}$

'Département de Biologie, Faculté des Sciences, Université Badji Mokhtar Annaba, Algérie. Email: tarek_hamel@yahoo.fr 'Laboratoire d'écopédologie, Département de Biologie, Faculté des Sciences, Université Badji Mokhtar Annaba, Algérie

\section{Correspondencia}

H. Tarek

e-mail: tarek_hamel@yahoo.fr

Recibido: 28 mayo 2018

Aceptado: 1 julio 2018

Publicado on-line: octubre 2018

\begin{abstract}
Résumé
Cette étude rapporte des données sur la diversité des Ptéridophytes du Parc National d'El Kala (Nord-Est algérien). Treize stations à caractéristiques écologiques différentes ont été prospectées; 27 espèces on été identifiés, dont 7 sont rares. L'inventaire de ces taxons constitue une étape importante pour le développement des stratégies de conservation des ressources génétiques et de la diversité des fougères et lycophytes sur l'ensemble de leur aire de distribution naturelle. Le statut des espèces et les principales menaces pour les habitats sont discutés.
\end{abstract}

Mots clés: Fougères, Ptéridophytes, Algérie, menaces, conservation.

\begin{abstract}
Inventory of pteridophytes in the El Kala National Park (Eastern Algeria)

This study reports data on the diversity of the Pteridophyte of El Kala National Park (North east Algeria). Thirteen stations with different ecological characteristics were prospected; 27 species have been identified (7 of which are exceptionally rare).

The inventory of these species constitutes an important stage for the development of the strategies of preservation of the genetic resources and the diversity of ferns and lycophytes on their whole area of natural distribution. The status of species and major threats to the habitats are discussed.
\end{abstract}

Key words: Ferns, Pteridophytes, Algeria, threats, conservation.

\section{Introduction}

Les ptéridophytes comprennent deux lignées évolutives distinctes et non étroitement apparentées, les lycophytes et les fougères parfois nommées monilophytes (Pryer et al. 2004; Smith etal. 2006; PPG1 2016). Ces lignées d'origine plus anciennes que celles des plantes à graines, continuent d'occuper de nombreuses niches écologiques diverses, terrestres, marécageuses et même aquatiques.

La zone de biodiversité de l'Afromontagne de l'Est, est classée comme une zone de l'hyperdiversité ou point chaud ('hotspot') (Myers et al. 2000; Mittermeier et al. 2011). Sur 11916 espèces de ptéridophytes connues dans le monde (PPG1 2016), l'Algérie abrite 82 espèces (Dobignard \&
Chatelain, 2010).

Selon Quézel et Santa (1962), l'Algérie est découpée en 20 secteurs phytogéographiques, dont 15 pour l'Algérie du Nord. Les secteurs les plus riches en ptéridophytes sont K1 (33 taxons) et K3 (28 taxons). Ensuite, viennent d'autres secteurs : K2 et A1 (avec 27 taxons pour chacun), A2 (avec 25 taxons) et $\mathrm{O} 1$ (avec 23 taxons).

Cette richesse remarquable en fougères dans la Numidie K3 s'explique que les plaines et reliefs littoraux de cette zone sont partie prenante du point-chaud de biodiversité végétale nommé "Kabylies-Numidie-Kroumirie" (Véla \& Benhouhou, 2007).

Le vaste ensemble de point-chauds renferme le site d'El Kala1. II est considéré comme une Zone Importante pour les Plantes (Important Plants 
Area) dans le sud de la Méditerranée en raison de sa haute valeur patrimoniale en termes d'espèces végétales menacées et/ou localement endémiques ou rares (Yahi et al., 2012).

À cet égard, cette contribution vise à fournir de nouvelles connaissances sur les ptéridophytes du Parc National d' El Kala.

\section{Présentation de la zone d'étude}

La présente étude a pour cadre le Parc National d'El Kala (PNEK), qui occupe une superficie de 78438 ha (Fig. 1). Elle est caractérisée par une grande diversité de paysages, ainsi le patrimoine biologique de la région demeure l'un des plus diversifiés du bassin méditerranéen occidental (Stevenson et al., 1988 ; Brahmia et al., 2002).

Comme l'indique la figure 1, le PNEK est localisé entre $36^{\circ} 55^{\prime}$ et $36^{\circ} 90^{\prime} \mathrm{N}$ et $08^{\circ} 16^{\prime}$ et $08^{\circ} 43^{\prime} \mathrm{E}$. II est limité au Nord par la mer Méditerranée, à l'Est par la frontière Algéro-Tunisienne, à l'Ouest par les plaines d'Annaba et au Sud par les monts de la Medjerda (Grimes, 2005).

D'après le climagramme d'Emberger (1955), la région d'El Kala est localisée dans l'étage bioclimatique sub-humide à hiver chaud, à la limite de l'étage humide.

\section{Méthodes}

La liste des espèces a été établie selon un échantillonnage systématique pendant les printemps et les automnes des années (20102016) et à partir de relevés floristiques effectués au niveau des groupements végétaux de chêne liège (Quercus suber L.), de maquis à oléo-lentisque (Olea europaea L. et Pistacia lentiscus L.), des

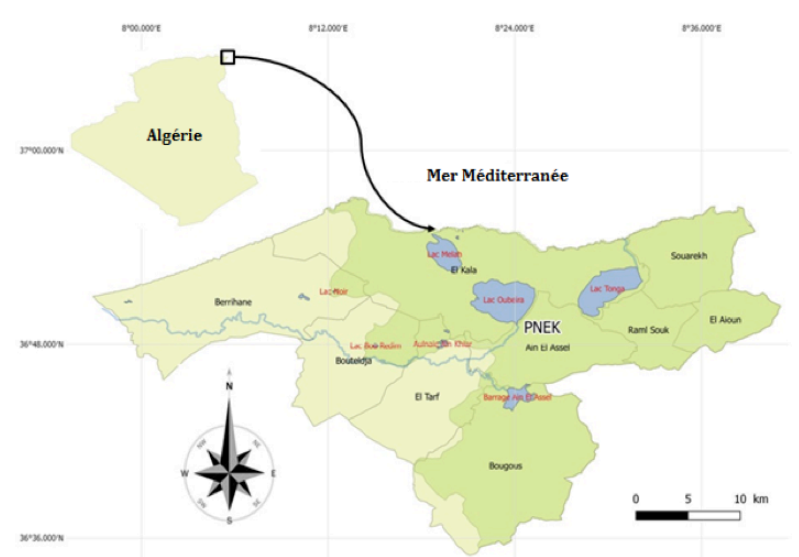

Figure 1. Situation de la région d'étude en Algérie : le Parc National d'El Kala.

Figure 1. Location of the study area in Algeria: El Kala National Park. ripisylves (Alnus glutinosa (L.) Gaertn./ Nerium oleander $\mathrm{L}$.), des prairies humides et des zones lacustres. La surface du relève doit être au moins égale à l'aire minimale, contenant la quasi-totalité des espèces présentes (Braun-Blanquet, 1932).

Les taxons ont été identifiés selon les flores de Quézel \& Santa (1962), Maire (1952) et Prelli (2001). La taxonomie a toutefois été mise à jour pour les espèces inventoriées en tenant compte des travaux récents compilés dans l'index synonymique et bibliographique de la flore d'Afrique du Nord (Dobignard \& Chatelain, 2010), le site web de la base de données des plantes d'Afrique [http:// www.ville-ge.ch/ musinfo/bd/cjb/africa/ recherche. php?langue $=\mathrm{fr}$ ] et la classification consensuelle récemment établie par le Pteridophyte Phylogeny Group (PPG1 2016). Les spécimens d'herbier collectés ont été identifiés puis déposés au niveau de l'herbier du Département de Biologie de l'Université Badji Mokhtar-Annaba, Algérie.

Nous avons organisé les taxons recensés par famille et les espèces sont données dans l'ordre alphabétique.

Les espèces recensées ont été renseignées par leur statut biogéographique d'après Pignatti (1982), Blanca et al. (2009), et leur type biologique selon Raunkiaer (1934), Pignatti (1982) et Blanca et al. (2009).

La liste rouge produite permet de mettre en évidence les taxons les plus menacés et de proposer des priorités dans les politiques de sauvegarde et de conservation de la biodiversité végétale. Nous avons aussi considéré comme espèces d'intérêt patrimonial les espèces protégées par le Décret $n^{\circ}$ 03-12/12-28 complétant la liste des espèces végétales non cultivées et protégées en Algérie (J.O.R.A. 2012).

L'ensemble des relevés floristiques a été soumis à deux analyses : une Analyse Canonique des Correspondances (ACC) et une Analyse Linéaire Discriminante (ALD).

Le croisement des données de la flore avec les variables environnementales (altitude, substrat, exposition, pente, taux de recouvrements des ligneux et des herbacés "tableaux 1») est obtenu par l'analyse canonique des correspondances (ACC). Le nuage résultant de l'ACC permet de visualiser le pourcentage explicatif d'une variable sur une autre (Ter Braak, 1995).

Ensuite le tableau des stations a été soumise à I'Analyse Linéaire Discriminante (ALD) (Legendre \& Legendre, 2012) afin d'obtenir une ségrégation des principaux groupes des stations des fougères de la région d'étude.

Ces deux analyses sont été effectuées en utilisant le langage informatique $R$, version 3.0.1 (R 


\begin{tabular}{|c|c|c|c|c|c|c|c|}
\hline Code & Station & Biotope & $\begin{array}{l}\text { Altitude } \\
\text { (m) }\end{array}$ & $\begin{array}{l}\text { Cordonnées de } \\
\text { GPS }\end{array}$ & $\begin{array}{l}\text { Roche mère selon Vila } \\
(1980)\end{array}$ & $\begin{array}{l}\text { Pente } \\
\%\end{array}$ & $\begin{array}{c}\text { Nombre } \\
\text { de taxons } \\
\text { observés }\end{array}$ \\
\hline $\mathrm{R} 1$ & Bougous & Subéraie & 482 & $\begin{array}{l}36^{\circ} 42^{\prime} 7.10^{\prime \prime} \mathrm{N} \\
8^{\circ} 27^{\prime} 36.377^{\prime \prime} \mathrm{E}\end{array}$ & Grès numidiens & 25 & 7 \\
\hline $\mathrm{R} 2$ & Cap Segleb & Maquis & 47 & $\begin{array}{l}36^{\circ} 56^{\prime} 20.28^{\prime \prime} \mathrm{N} ; \\
8^{\circ} 36^{\prime} 29.94^{\prime \prime} \mathrm{E}\end{array}$ & Grès numidiens & $<10$ & 8 \\
\hline R3 & $\begin{array}{l}\text { Arboretum } \\
\text { Tonga }\end{array}$ & $\begin{array}{l}\text { Prairie } \\
\text { humide }\end{array}$ & 8 & $\begin{array}{l}36^{\circ} 52^{\prime} 46.11^{\prime \prime} \mathrm{N} ; \\
8^{\circ} 31^{\prime} 19.48 \text { ' }\end{array}$ & Quaternaire indifférencie & $<5$ & 6 \\
\hline $\mathrm{R} 4$ & $\begin{array}{l}\text { Djebel El } \\
\text { Korsi }\end{array}$ & Subéraie & 391 & $\begin{array}{l}36^{\circ} 52 \text { '35.81"N ; } \\
8^{\circ} 15 \text { '37.01"E }\end{array}$ & Grès numidiens & 20 & 7 \\
\hline R5 & Lac Bleu & $\begin{array}{l}\text { Zone } \\
\text { lacustre }\end{array}$ & 6 & $\begin{array}{l}36^{\circ} 54^{\prime} 39.45^{\prime \prime} \mathrm{N} ; \\
8^{\circ} 20^{\prime} 13.88^{\prime \prime} \mathrm{E}\end{array}$ & Quaternaire indifférencie & $<5$ & 4 \\
\hline R6 & Lac Oubeira & $\begin{array}{l}\text { Zone } \\
\text { lacustre }\end{array}$ & 61 & $\begin{array}{l}36^{\circ} 52^{\prime} 10.29 " \mathrm{~N} ; \\
8^{\circ} 22^{\prime} 41.99^{\prime \prime} \mathrm{E}\end{array}$ & Quaternaire indifférencie & $<5$ & 4 \\
\hline $\mathrm{R} 7$ & $\begin{array}{l}\text { Oued Bou- } \\
\text { gous }\end{array}$ & Aulnaie & 740 & $\begin{array}{l}36^{\circ} 40^{\prime} 54.48 ” \mathrm{~N} ; \\
8^{\circ} 26^{\prime} 50.97^{\prime \prime} \mathrm{E}\end{array}$ & Grès numidiens & 35 & 10 \\
\hline $\mathrm{R} 8$ & Ksar Fatma & Subéraie & 405 & $\begin{array}{l}36^{\circ} 49^{\prime} 40.89^{\prime \prime} \mathrm{N} ; \\
8^{\circ} 39^{\prime} 53.87^{\prime \prime} \mathrm{E}\end{array}$ & Grès numidiens & 22 & 9 \\
\hline R9 & Souarekh & Maquis & 178 & $\begin{array}{l}36^{\circ} 52^{\prime} 42.53^{\prime \prime} \mathrm{N} ; \\
8^{\circ} 35^{\prime} 3.32 \prime \mathrm{E}\end{array}$ & Quaternaire indifférencie & $<5$ & 3 \\
\hline $\mathrm{R} 10$ & Ain Khiar & Aulnaie & 33 & $\begin{array}{l}36^{\circ} 48^{\prime} 56.41 \text { "N ; } \\
8^{\circ} 20^{\prime} 26.71^{\prime \prime} \mathrm{E}\end{array}$ & Quaternaire indifférencie & $<5$ & 12 \\
\hline $\mathrm{R} 11$ & Bourdim & Aulnaie & 57 & $\begin{array}{l}36^{\circ} 48^{\prime} 50.59 " \mathrm{~N} ; \\
8^{\circ} 20^{\prime} 36.47^{\prime \prime} \mathrm{E}\end{array}$ & Quaternaire indifférencie & $<5$ & 7 \\
\hline $\mathrm{R} 12$ & Oued Nhal & Ripisylve & 5 & $\begin{array}{l}36^{\circ} 56^{\prime} 2.26^{\prime \prime} \mathrm{N} \\
8^{\circ} 14^{\prime} 24.42^{\prime \prime} \mathrm{E}\end{array}$ & Quaternaire indifférencie & $<5$ & 6 \\
\hline $\mathrm{R} 13$ & El Henia & Maquis & 70 & $\begin{array}{l}36^{\circ} 51^{\prime} 34.44 ” \mathrm{~N} \\
8^{\circ} 14^{\prime} 38.799^{\prime \prime} \mathrm{E}\end{array}$ & Quaternaire indifférencie & $<5$ & 4 \\
\hline
\end{tabular}

Tableau 1. Situation géographique des stations échantillonnées dans le PNEK. Table 1. Geographical location of sampled stations in EKNP.

Core Team, 2013) et une analyse multivariée sur le package « ade4 » disponible dans R (Chessel et al., 2004).

\section{Résultats}

\section{Diversité ptéridologique}

$\mathrm{Au}$ total 27 espèces de ptéridophytes appartenant à 16 familles ont été identifiées dans la région d`étude (Tableau 2).

Ces espèces se répartissent en 17 genres (Adiantum, Anogramma, Asplenium, Athyrium, Cheilanthes, Cystopteris, Equisetum, Isoëtes, Marsilea, Ophioglossum, Osmunda, Polypodium, Polystichum, Pteridium, Salvinia, Selaginella et Thelypteris), avec le genre Asplenium le plus diversifié (8 taxons).

Parmi ces 27 espèces, 7 ont été observées dans une seule station (Asplenium billotii F. W. Schultz, Asplenium sagittatum (DC.) A.J.
Bange, Asplenium scolopendrium L., Marsilea minuta L., Ophioglossum lusitanicum L., Cheilanthes maderensis Lowe, Cystopteris fragilis subsp. diaphana (Bory) Litard.).

En revanche, certaines espèces ont une répartition très large dans la région d'étude (Anogramma leptophylla (L.) Link, Pteridium aquilinum (L.) Kuhn et Polypodium cambricum L. subsp. cambricum).

La répartition des types biologiques dans la flore étudiée suit, dans l'ensemble, le schéma suivant : hémicryptophyte rosale (17 taxons), géophyte à rhizome (3 taxons), hémicryptophyte à rhizome, hémicryptophyte cespiteuse et thérophyte rosale (2 taxons pour chacun), chamaephyte rampante (1 taxon).

Sur le plan biogéographique, la flore étudiée est caractérisée par le type: subcosmopolite (7 taxons), méditerranéen ( 5 taxons), euro -méditerranéen et méditerranéen atlantique (4 taxons pour chacun), 


\begin{tabular}{|c|c|c|c|}
\hline Famille & Taxon & Type biologique & Type biogéographique \\
\hline \multirow[t]{8}{*}{ Aspleniaceae } & Asplenium adiantum-nigrum $\mathrm{L}$. & Hém. ros. & Euro -méditerranéen \\
\hline & Asplenium billotii F. W. Schultz & Hém. ros. & Euro -méditerranéen \\
\hline & Asplenium ceterach $\mathrm{L}$. & Hém. ros. & Eurasien méditerranéen \\
\hline & Asplenium obovatum Viv. subsp. obovatum & Hém. rhiz. & Sténo méditerranéen \\
\hline & Asplenium onopteris L. & Hém. ros. & Euro - méditerranéen \\
\hline & $\begin{array}{l}\text { Asplenium trichomanes L. subsp. quadrivalens } \\
\text { D. E. Meyer }\end{array}$ & Hém. ros. & Subcosmopolite \\
\hline & Asplenium sagittatum (DC.) A.J. Bange & Hém. ros. & Méditerranéen \\
\hline & Asplenium scolopendrium $\mathrm{L}$. & Hém. ros. & Euro - méditerranéen \\
\hline Athyriaceae & Athyrium filix-femina (L.) Roth & Hém. ros. & Holarctique \\
\hline \multirow[t]{2}{*}{ Cystopteridaceae } & $\begin{array}{l}\text { Cystopteris fragilis subsp. diaphana (Bory) Lit- } \\
\text { ard. }\end{array}$ & Hém. ros. & Subtropical \\
\hline & Cystopteris fragilis (L.) Bernh. subsp. fragilis & Hém. ros. & Subcosmopolite \\
\hline Dennstaedtiaceae & Pteridium aquilinum (L.) Kuhn & Géo. rhiz. & Subcosmopolite \\
\hline Dryopteridaceae & $\begin{array}{l}\text { Polystichum setiferum (Forsskål) T. Moore ex } \\
\text { Woynar }\end{array}$ & Hém. ros. & Méditerranéen atlantique \\
\hline \multirow[t]{2}{*}{ Equisetaceae } & $\begin{array}{l}\text { Equisetum ramosissimum Desf. } \\
\text { subsp. ramosissimum }\end{array}$ & Hém. ces. & Tropical \\
\hline & Equisetum telmateia Ehrh & Hém. ces. & Holarctique \\
\hline \multirow[t]{2}{*}{ Isoëtaceae } & Isoëtes vetata $\mathrm{A}$. Braun & Hém. ros. & Méditerranéen atlantique \\
\hline & Isoëtes histrix Bory & Hém. ros & Méditerranéen atlantique \\
\hline Marsileaceae & Marsilea minuta L. & Géo. rhiz. & Tropical \\
\hline Ophioglossaceae & Ophioglossum lusitanicum L. & Géo. rhiz. & Méditerranéen \\
\hline Osmundaceae & Osmunda regalis L. & Hém. ros. & Subcosmopolite \\
\hline Polypodiaceae & Polypodium cambricum L. subsp. cambricum & Hém. ces. & Méditerranéen atlantique \\
\hline \multirow[t]{2}{*}{ Pteridaceae } & Adiantum capillus-veneris L. & Hém. rhiz. & Subcosmopolite \\
\hline & Anogramma leptophylla (L.) Link & Th. ros. & Subcosmopolite \\
\hline Salviniaceae & Salvinia natans (L.) All. & Th. ros. & Eurasien tempéré \\
\hline Selaginellaceae & Selaginella denticulata (L.) Spring & Ch. ram. & Méditerranéen \\
\hline Sinopteridaceae & Cheilanthes maderensis Lowe & Hém. ros. & Méditerranéen \\
\hline Thelypteridaceae & Thelypteris palustris Schott & Hém. ros. & Subcosmopolite \\
\hline
\end{tabular}

Tableau 2. Type biologique et biogéographique des ptéridophytes observées dans la région d'étude.

Table 2. Biological and biogeographic type of pteridophytes observed in the study area.

tropical (3 taxons), holarctique et eurasien (s.I.) (2 taxons pour chacun).

\section{Analyse canonique des correspondances flore ptéridologique / environnement}

La carte de l'analyse canonique des correspondances (Fig. 3), associé la répartition des stations de fougères aux variables du milieu et à la typologie de la végétation. Le plan formé par le premier et le second axe totalise un taux d'inertie de $67,20 \%$.

Au vue de ces observations, beaucoup de stations ont révélé un nombre très important des espèces. Quatre stations sont relativement riches en espèces (Ain Khiar avec 12 espèces, Ksar Fatma avec 10 espèces, Oued Bougous avec 9 espèces et Cap Segleb avec 8 espèces).

La station ( $R 7$ ) apparaît isolée, en rapport avec sa situation altitudinale extrême (740 m).

Les trois stations (R1), (R4) et 

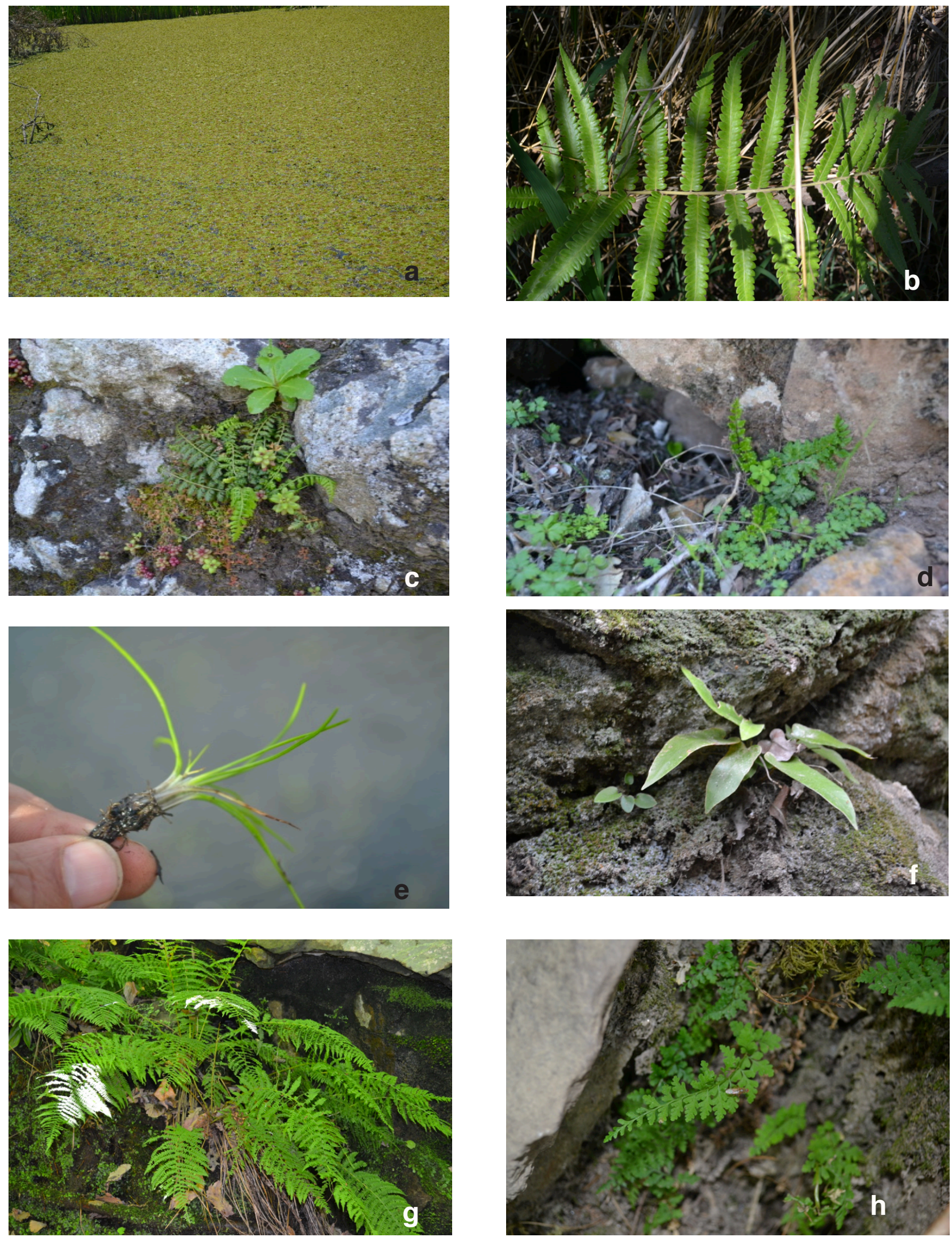

Figure 2. 

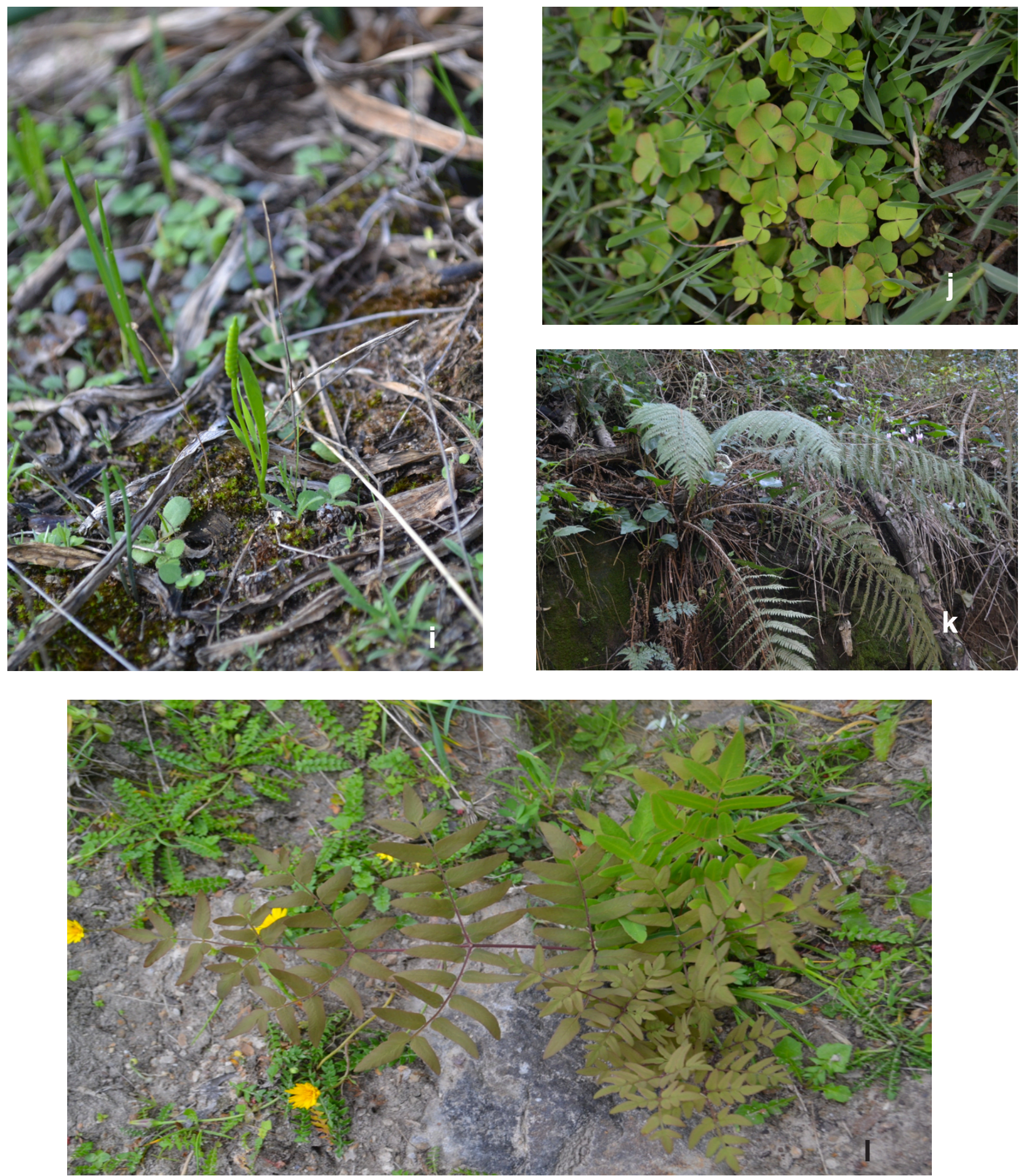

Figure 2 (continuation). Les ptéridophytes remarquables dans la région d'étude (a. Salvinia natans (L.) All., b. Thelypteris palustris Schott, c. Asplenium obovatum Viv. subsp. obovatum, d. Cystopteris fragilis (L.) Bernh. subsp. fragilis, e. Isoëtes histrix Bory, f. Phyllitis sagittata (DC.) Guinea \& Heywood, g. Athyrium filix-femina (L.) Roth, h. Cystopteris fragilis subsp. diaphana (Bory) Litard., i. Ophioglossum lusitanicum L., j. Marsilea minuta L., k. Polystichum setiferum (Forsskål) T. Moore ex Woynar, I. Osmunda regalis L.

Figure 2. The remarkable pteridophytes in the study area. 


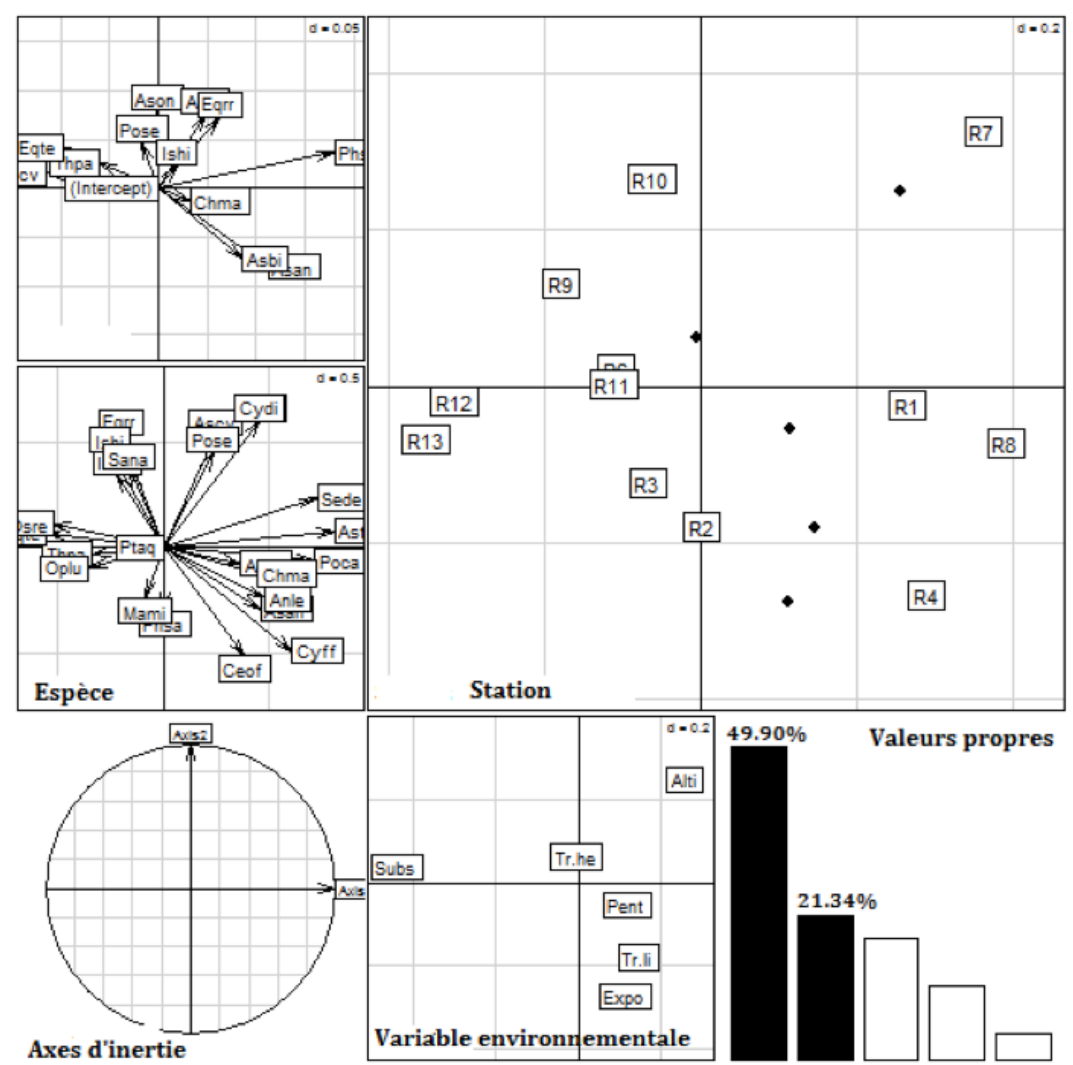

Figure 3. Carte de l'analyse canonique des correspondances 13 stations $X 27$ espèces X 6 variables environnementales.

Figure 3. Map of the canonical correspondence analysis 13 stations $X 27$ species $X 6$ environmental variables.

apparaissent dans la partie positive de l'axe 2, en relation avec leur exposition nord, la pente plutôt faible. Ces stations sont également caractérisées par un recouvrement des ligneux dominé par la présence de chêne liège (Quercus suber L.), avec des sous bois dense d'Erica arborea L., Cytisus villosus Pourret, Phillyrea latifolia L., Arbutus unedo L., Calicotome villosa Poiret Link subsp. villosa et de Cistus salvifolius L.

Sur la partie négative de l'axe 2, les stations R2, R3, R6, R9, R10, R11, R12 et R13 sont situées en basses altitudes et se répartissent selon un gradient de substrat.

Alors les stations (R12 et R13), qui sont installées sur un sol sableux, présentent un très faible recouvrement de herbacées. Notons que le reste de ces stations, situées sur substrat de grès et d'argile de Numidie, sont assez fortement colonisées par des herbacées; les stations possèdent un faible recouvrement des ligneux non négligeable.

\section{Typologie des stations de fougères}

L'ALD réalisée sur l'ensemble des 13 stations et les 6 variables environnementales mette en évidence l'existence d'un gradient d'altitude dans le sens de l'axe 1, entre les milieux ouverts et milieux fermés (Fig. 4). Cette analyse est décrite par les deux premiers facteurs ( $F 1=45,58 \%$, $\mathrm{F} 2=27,83 \%)$. Trois principaux groupes peuvent ainsi être distingués :

Le groupe «1» est caractérisé par les deux stations R2 et R12. Ce groupe est localisé aux basses altitudes (5- $47 \mathrm{~m})$. Ses stations correspondent à des formations de maquis bas de Cap Segleb (R2) à Quercus coccifera L. et Juniperus oxycedrus subsp. macrocarpa (Sm.) Neilr, et une ripisylve de Oued Nhal à Nerium oleander L. et Populus alba L. sur le maquis bas de Cap Rosa.

Le groupe «2» est caractérisé par les stations (R3, R5, R6, R9, R10, R11 et R13). Ce groupe occupe une tranche altitudinale allant des basses aux moyennes altitudes (6 - $187 \mathrm{~m})$, avec des formations herbacées très denses, et révèlent aussi l'importance du substrat de grès et argile de Numidie et quaternaire indifférencié ce qui permet l'observation des grandes taches de fougères sur le terrain, avec une ptéridoflore très diversifié (le cas de la station Ain Khiar avec 12 espèces). 


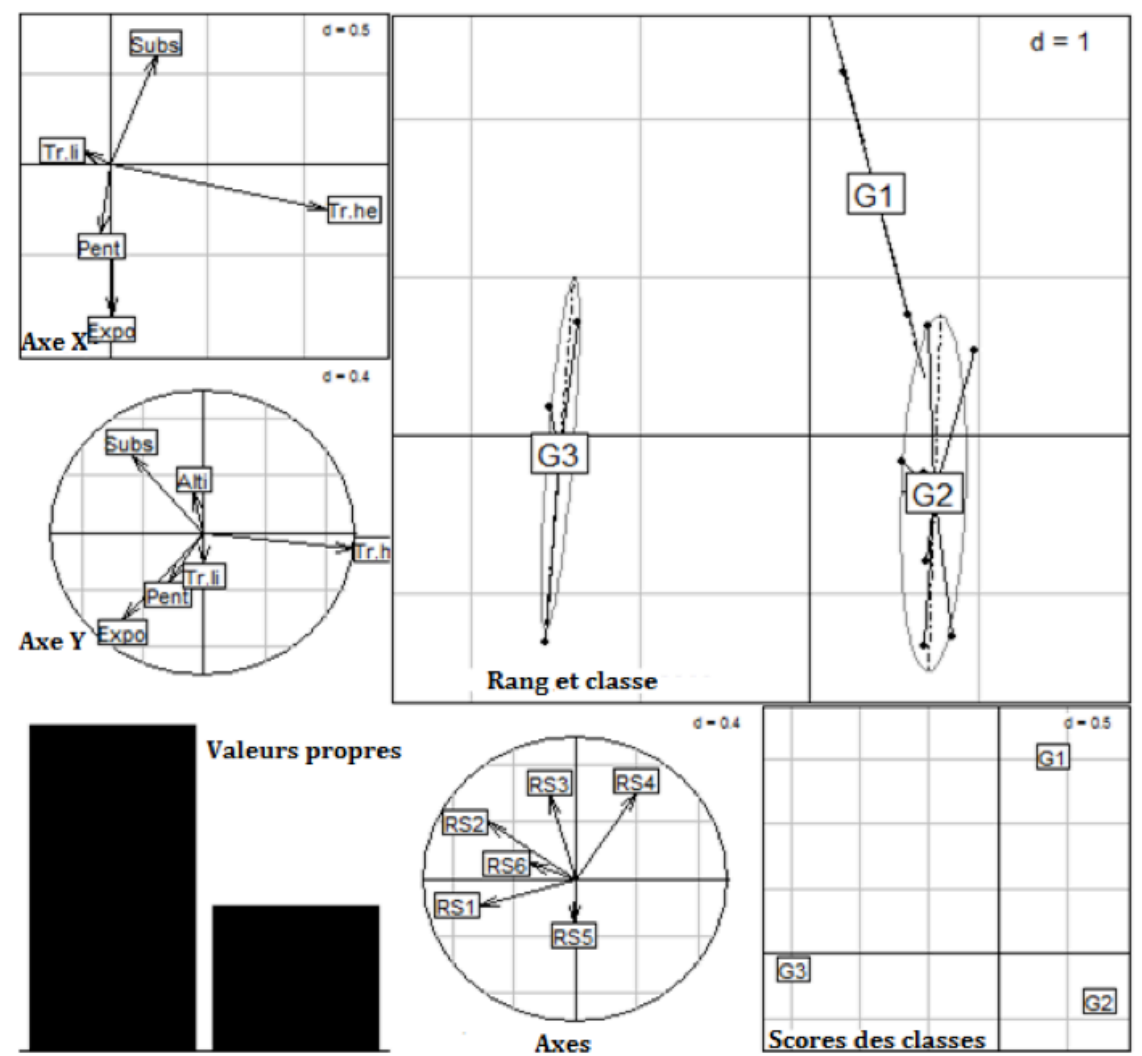

Figure 4. Analyse Linéaire Discriminante sur les assemblages des 13 stations $X 6$ variables environnementales.

Figure 4. Linear Discriminant Analysis on the Assemblies of the 13 Stations X 6 Environmental Variables.

Sur le coté positif de l'axe 2, le groupe «3» est constitué par quatre stations (R1, R4, R7 et R8)

II occupe essentiellement les plus hautes altitudes (391 à 740 m) et se caractérise par des formations sylvatiques plus ou moins fermées. II se rapporte notamment aux forêts denses de chêne liège (R1 et R4), de maquis à oléo-lentisque d'altitude avec le mélange de chêne liège (R8) et de ripisylve à Alnus glutinosa (L.) Gaertn. Les espèces caractéristiques de ce groupement sont fréquentes en haute altitude dans les formations forestières plus ou moins fermées. Ces stations sont caractérisées par des pentes de 20 à 35\% et de substrat typiquement de grès et argile de Numidie.

\section{Discussion}

La ptéridoflore du Parc National d'El Kala est relativement diversifiée, puisque 27 espèces ont été observées, avec la dominance de la famille des Aspleniaceae.

Cette famille est aussi la plus diversifiée à l'échelle du territoire algérien (Quézel \& Santa, 1962). Ces résultats, que ce soit pour les genres ou les espèces, confirment les observations réalisées par Louhi-Haou (2014) ; Hamel et al. (2017 a \& b) sur la ptéridoflore de la Numidie.

En se basant sur les travaux de Quézel \& Santa (1962), actualisés pour la taxonomie suivant PPG 1 (2016) (Tableau 3), on remarque que cette ptéridoflore présente huit taxons communs, sept assez communs, et quatre assez rares. Cependant, les taxons rares s'élèvent à quatre aux quels s'ajoutent deux très rares et un rarissime, soit en tout $25 \%$ de l'ensemble des ptéridophytes du Parc National d'El Kala. Une hypothèse est que cette rareté est la conséquence d'actions directes et/ou indirectes des dégradations que subissent les habitats correspondants.

Une seule espèce est évaluée «vulnérable» (V) dans l'ancienne liste rouge de 1997 (Walter \& Gillet, 1998), mais n'a pas encore fait l'objet d'une évaluation selon les nouveaux critères (UICN 2017). Dans cette dernière liste, sept taxons sont évalués dans la catégorie de préoccupation mineure (LC). En outre, 4 taxons figurent sur la liste des espèces végétales non cultivées et protégées qui en comporte 449 (JORA., 2012).

Les espèces appartenant à l'ensemble subcosmopolite sont au nombre de 7 , soit 25,92 $\%$ de la flore répertoriée. Ce pourcentage est 


\begin{tabular}{|c|c|c|c|c|}
\hline Famille & Taxon & Rareté & JORA (2012) & $\begin{array}{l}\text { UICN } 2017 \\
\text { (Walter \& Gillet, } \\
\text { 1998) }\end{array}$ \\
\hline \multirow[t]{8}{*}{ Aspleniaceae } & Asplenium adiantum-nigrum L. & $\mathrm{C}$ & & \\
\hline & Asplenium billotii F. W. Schultz & AR & & \\
\hline & Asplenium ceterach $\mathrm{L}$. & $\mathrm{C}$ & & \\
\hline & Asplenium obovatum Viv. subsp. obovatum & AR & & \\
\hline & Asplenium onopteris L. & $\mathrm{C}$ & & \\
\hline & $\begin{array}{l}\text { Asplenium trichomanes L. subsp. quadrivalens } \\
\text { D. E. Meyer }\end{array}$ & C & & \\
\hline & Asplenium sagittatum (DC.) A.J. Bange & AR & & \\
\hline & Asplenium scolopendrium L. & $A C$ & & V \\
\hline Athyriaceae & Athyrium filix-femina (L.) Roth & AR & & \\
\hline \multirow[t]{2}{*}{ Cystopteridaceae } & $\begin{array}{l}\text { Cystopteris fragilis subsp. diaphana (Bory) Li- } \\
\text { tard. }\end{array}$ & $\mathrm{R}$ & & \\
\hline & Cystopteris fragilis (L.) Bernh. subsp. fragilis & $\mathrm{R}$ & & \\
\hline Dennstaedtiaceae & Pteridium aquilinum (L.) Kuhn & $\mathrm{C}$ & & \\
\hline Dryopteridaceae & $\begin{array}{l}\text { Polystichum setiferum (Forsskål) } \\
\text { T. Moore ex Woynar }\end{array}$ & $\mathrm{R}$ & & \\
\hline \multirow[t]{2}{*}{ Equisetaceae } & $\begin{array}{l}\text { Equisetum ramosissimum Desf. } \\
\text { subsp. ramosissimum }\end{array}$ & $\mathrm{C}$ & & \\
\hline & Equisetum telmateia Ehrh & $A C$ & & LC \\
\hline \multirow[t]{2}{*}{ Isoëtaceae } & Isoëtes vetata $\mathrm{A}$. Braun & $A C$ & & \\
\hline & Isoëtes histrix Bory & $A C$ & & \\
\hline Marsileaceae & Marsilea minuta L. & RRR & $\mathrm{P}$ & \\
\hline Ophioglossaceae & Ophioglossum lusitanicum L. & $\mathrm{R}$ & $\mathrm{P}$ & LC \\
\hline Osmundaceae & Osmunda regalis $\mathrm{L}$. & C & & LC \\
\hline Polypodiaceae & Polypodium cambricum L. subsp. cambricum & $\mathrm{AC}$ & & \\
\hline \multirow[t]{2}{*}{ Pteridaceae } & Adiantum capillus-veneris L. & $\mathrm{AC}$ & & LC \\
\hline & Anogramma leptophylla (L.) Link & C & & \\
\hline Salviniaceae & Salvinia natans (L.) All. & $\mathrm{RR}$ & $\mathrm{P}$ & LC \\
\hline Selaginellaceae & Selaginella denticulata (L.) Spring & $A C$ & & LC \\
\hline Sinopteridaceae & Cheilanthes maderensis Lowe & AR & & \\
\hline Thelypteridaceae & Thelypteris palustris Schott & $\mathrm{RR}$ & $\mathrm{P}$ & LC \\
\hline
\end{tabular}

Tableau 3. Valeur patrimoniale des ptéridophytes de la région d'étude: $\mathrm{P}$ (Protégée), V (Vulnérable), LC (Préoccupation mineure), C (Commun), A (Assez), R (Rare), RR (Très rare), RRR (Rarissime).

Table 3. Heritage value of pteridophytes in the study area.

comparable à celui donné par Hamel et al. (2017a \& b) pour la ptéridoflore de la région de Skikda (32\%) et de la péninsule de l'Edough (27,27\%).

La flore étudiée est dominée par les hémicryptophytes rosales (59,25\%). D'après Kazi Tani et al. (2010), il semblerait que la pluviosité, la faiblesse des éclairements lumineux et les pâturages des sous-bois favorisent le développement des hémicryptophytes. Barbéro et al. (2001) signalent que leur abondance dans les pays du Maghreb est due à la présence de la matière organique et de l'humidité. Cette richesse en hémicryptophytes peut s'expliquer aussi par l'importance des mycorhizes dans le sol (Boudiaf et al., 2014).

\section{Ecologie des ptéridophytes observées}

Sur le plan écologique, la majorité des espèces inventoriées tend à coloniser les milieux humides de notre région, en appréciant notamment l'ombre des arbres pour se développer.

La distribution des différentes espèces varie 
selon les milieux, les différents taxons ont été recensés dans la partie nord du Parc National d'El Kala, caractérisée par un substrat sablo-argilogréseux calcaire, un bioclimat humide à subhumide et un recouvrement des ligneux localement très abondant.

Les fougères forestières Athyrium filix-femina (L.) Roth et Cystopteris fragilis subsp. diaphana (Bory) Litard. colonisent nettement la station de Oued Bougous. Cette région montagneuse est caractérisée par un relief à forte pente. Notons que les couches intercalées de grès et diargile de Numidie sont favorables au développement de chênaie (Slimani et al., 2013).

Quant aux espèces à valeur patrimoniale (rares à l'échelle nationale et/ou menacées au niveau mondial), deux sont associées aux prairies humides des mares et des lacs (Thelypteris palustris Schott, Marsilea minuta L.), et deux sont associées aux ripisylves de moyenne altitude sur les reliefs (Polystichum setiferum (Forsskål) T. Moore ex Woynar et Salvinia natans (L.) All.). Bien qu'en Algérie, c'est surtout dans les milieux humides que les raréfactions ou disparitions d'espèces sont les plus nombreuses (Mathez et al., 1985 ; Hamel et al., 2013).

Enfin, deux espèces à valeur patrimoniale sont associées aux maquis ouverts méditerranéens de basse altitude (Cystopteris fragilis (L.) Bernh. subsp. fragilis, Ophioglossum lusitanicum L.).

\section{Diversité ptéridologique en Algérie}

En Algérie, près de 82 taxons ont été observés (Dobignard \& Chatelain, 2010), sur un total de 99 recensés en Afrique du nord (Meddour, 2008). Sur l'ensemble de ces taxons, une vingtaine sont jugés rares et se retrouvent uniquement dans des milieux forestiers et humides du secteur Kabylo-Numidien. S'agissant des espèces, autrefois signalées dans les dayas et mares des secteurs algérois, leur disparition est plus que probable, ce qui est imputé à la destruction des habitats (Le Houérou, 1995).

Dans le secteur numidien du nord-est algérien (regroupant trois régions et englobant la nôtre), Haou et al. (2011) ont inventorié 20 espèces/sousespèces de fougères, soit seulement 7 de moins que dans la présente étude (Tableau 4). Au sein de se secteur, la péninsule de l'Edough abrite 22 taxons (Hamel et al., 2017 b). Ainsi la région de Skikda est la plus pauvre avec 16 espèces (Hamel et al., 2017 a).

À superficie trois fois plus grande que le secteur numidien, la région de Tlemcen (O3) renferme 26 espèces/sous-espèces (Medjahdi et al., 2013). Par ailleurs, le Parc National de Taza (K2) abrite 19 taxons sur une superficie de 3807 hectares.
La présente étude recense le plus d'espèces avec 27. Cette richesse est vraisemblablement due à l'association de la diversité géomorphologique (plissements du relief et affleurements géologiques) et de la zone de contact bioclimatique (climat subhumide au nord et humide à hyper humide au sud), créant des zones de carrefours biogéographiques remarquables.

\section{Menaces et conservation}

Les facteurs tels que le changement climatique, l'urbanisation croissante, l'industrialisation, l'empiètement sur les terres forestières, les activités de développement non planifiées, la surexploitation des ressources naturelles constituent une menace majeure pour la survie des ptéridophytes (Dixit, 2000). La collecte à grande échelle des fougères des forêts par les visiteurs et la population locale à des fins d>ornement, à usage médicinal ou pendant les excursions augmente également la pression sur ces plantes.

En Numidie, l'agriculture et l'urbanisation ont détruit presque entièrement l'équilibre des aulnaies et des ripisylves (habitats pour Osmunda regalis L., Athyrium filix-femina (L.) Roth et les Equisetum) diminuant ainsi la densité de leur population allant jusqu'à leur disparition dans certaines stations (Louhi-Haou, 2014).

\section{Conclusion}

L'analyse de la ptéridoflore du Parc National d'El Kala révèle son originalité écologique et floristique, induite par les particularités orographiques et surtout climatiques de la zone. Ces conditions ont permis le développement et le maintien d'une flore riche et diversifiée (27 taxons). L'élément subcosmopolite est bien représenté avec près de $25,92 \%$ de la flore recensée. La forte pression anthropique, notamment l'agriculture et les incendies, rend hypothétique le maintien à long terme de cette flore. Les espèces les plus fragiles sont particulièrement celles qui se trouvent à la limite de leur aire de répartition, comme c'est le cas de la plupart des espèces relevant de l'ensemble subcosmopolite (Thelypteris palustris Schott, Osmunda regalis L.). Pour cette raison, elles pourraient être encore plus sensibles au réchauffement climatique global qui s'amorce. Ces espèces se localisent essentiellement au niveau des forêts denses des milieux humides (lac et aulnaie), d'où la nécessité de leur protection et de la préservation de leur habitat. La plupart de ces taxons sont rares ou très rares et méritent une protection sans laquelle ils seront menacés de disparition dans la région. La répartition de 


\begin{tabular}{|l|l|c|c|c|}
\hline Source & Région/secteur & Surface (ha) & $\begin{array}{l}\text { Nombre d'espèces par secteur } \\
\text { (Quézel \& Santa, 1962) }\end{array}$ & $\begin{array}{l}\text { Nombre de } \\
\text { taxons recensés }\end{array}$ \\
\hline Présente étude & PNEK (K3) & 78438 & 28 & 27 \\
\hline Hamel et al. (2017 a) & Skikda (K3) & 5200 & 28 & 22 \\
\hline Hamel et al. (2017 b) & $\begin{array}{l}\text { Péninsule de } \\
\text { l'Edough (K3) }\end{array}$ & 47350 & 28 & 20 \\
\hline Haou et al. (2011) & Numidie (K3) & $>280159$ & 28 & 19 \\
\hline $\begin{array}{l}\text { Benabbas \& Kacmi } \\
\text { (2017) }\end{array}$ & $\begin{array}{l}\text { Parc National de } \\
\text { Taza (K2) }\end{array}$ & 3807 & 21 & 26 \\
\hline Medjahdi et al. (2013) & Tlemcen (O3) & 906100 & 21 & 26 \\
\hline
\end{tabular}

Tableau 4. Diversité ptéridologique dans différentes régions d’Algérie [Secteurs biogéographiques selon la subdivision proposée par Quézel \& Santa (1962)].

Table 4. Pteridological diversity in different regions of Algeria [Biogeographic sectors according to the subdivision proposed by Quézel \& Santa (1962)].

certaines espèces (Marsilea minuta L., Thelypteris palustris Schott) en Algérie est limitée au Parc National d'El Kala, ce qui plaide pour l'attribution d'un statut particulier pour tout le secteur.

Durant la période d'échantillonnage, Asplenium marinum L. signalée pour la région n'a pas été observée (Maire, 1952). La recherche continue pour cette espèce.

\section{Références bibliographiques}

Barbéro, M., Loisel, R., Médail, F. \& Quézel, P. (2001). Signification biogéographique et biodiversité des forêts du bassin méditerranéen. Bocconea, 13, 11-25.

Benabbas, F. \& Kacmi, K. (2017). Inventaire préliminaire des fougères du Parc National de Taza (Jijel, Algérie). Thèse de Master en Science Naturelle de l'Environnement, Université Abderrahmane MIRABejaia, Algérie, 71p.

Blanca, G., Cabezudo, B., Cueto, M., Lopez, CF. \& Torres, CM. (2009). Flora Vascular de Andalucía Oriental. Tome 1. Consejería de Medio Ambiente. Junta de Andalucía. 436p.

Boudiaf, I., Le Roux, C., Baudoin, E., Galiana, A., Beddiar, A., Prin, Y. \& Duponnois R. (2014). Soil Bradyrhizobium population response to invasion of a natural Quercus suber forest by the introduced nitrogen-fixing tree Acacia mearnsii in El Kala National Park, Algeria. Soil Biology \& Biochemistry, 70, 162-165.

Brahmia, Z., Dziri, H., Benyacoub, S. \& Chabi, Y. (2002). Biology of shrike (Lanus senator) in north east of Algeria. Rev. Ecol. (Terre \& Vie), 62, 128-134.

Braun-Blanquet, J. (1932). Plant sociology: the study of plant communities. Mc Graw Hill, New York.

Chessel, D., Dufour, A.B. \& Thioulouse, J. (2004). The ade4 package-I-: One-table methods. $R$ News, 4: 5-10.

Dobignard, A. \& Chatelain, C. (2010). Index synonymique de la flore d'Afrique du Nord (1 vol). C.J.B.G, Genève, $178 p$.
Emberger, L., (1955). Une classification biogéographique des climats. Trav. Lab. G,ol. Bot. et Zool., Fac. Sc. Montpellier, 7, 1-43.

Grimes, S., (2005). Plan de gestion de l'aire marine du Parc National d'El Kala (Wilaya d'El Tarf). Projet Régional pour le développement d'aires marines et côtières protégées dans la région de la Méditerranée (UNEP-MedMPA). 126 p.

Hamel, T., Seridi, R., de Bélair, G., Slimani, A.R. \& Babali, B. (2013). Flore vasculaire rare et endémique de la péninsule de l'Edough (Nord-Est algérien). Revue Synthèse des Sciences et de la Technologie, 26, 65-74.

Hamel, T., Boulemtafes, A., Slimani, AR., Madoui, BEM. \& Drid, MD. (2017a). Diversity and ecology of Pteridophytes in the Skikda region (North East Algeria). International Research Journal of Biological Sciences, 6(3), 42-47.

Hamel, T., Slimani, AR., Madoui, BEM. \& Boulemtafes, A. (2017b). Pteridophytes of Edough peninsula (North East Algeria). International Journal of research in Ayareda and Pharmacy, 8 (1), 92-96.

Haou, H., de Bélair, G., Ronald, L. \& Viane, L. (2011). Inventory of the ferns (filicopsida) of Numidia's (NorthEastern Algeria), International Journal of Biodiversity and Conservation, 3(6), 206-223.

J.O.R.A., (2012). Décret exécutif du 18 janvier 2012, complétant la liste des espèces végétales non cultivées et protégées. Journal officiel de la république algérienne, $\mathrm{n}^{\circ}$ 3-12/12 du 18-01-2012.

Kazi Tani, C., Lebourgeois, T. \& Munoz, F. (2010). Aspects floristiques de la flore des champs du domaine phytogéographique oranais (Nord-Ouest algérien) et persistance d'espèces rares et endémiques. Flora Mediterranea, 20, 5-22.

Le Houerou, H.N. (1995). Bioclimatologie et biogéographie des steppes arides du Nord de l'Afrique. Options médit., série $B$ : études et recherches. $N^{\circ} 10,396$ p.

Legendre, P. \& Legendre, L. (2012). Numerical ecology, 3ème édition. Elsevier Science, Amsterdam. 1006p.

Louhi-Haou, S., (2014). Ecologie des ptéridophytes en 
Numidie (Nord Est de l'Algérie). Thèse de Doctorat en Ecologie végétale, université Badji Mokhtar Annaba, Algérie, $189 \mathrm{p}$.

Maire, R., (1952). Flore de l'Afrique du Nord (Maroc, Algérie, Tunisie, Tripolitaine, Cyrénaïque et Sahara). Lechevalier, Paris, $371 \mathrm{p}$.

Mathez, J., Quézel, P. \& Raynaud, C. (1985). The Maghreb countries. In : Gomez-Campo V., ed., Plant conservation in the Mediterranean area, 141-157. Junk Publ., Dordrecht.

Meddour, R. (2008). Taxinomie, chorologie et régression des ptéridophytes d'Algérie : Synthèse bibliographique. $28 \mathrm{p}$.

Medjahdi, B., Letreuch-Belarouci, A. \& Prelli, R. (2014). Actualisation du catalogue des ptéridophytes du nord ouest algérien (région de Tlemcen). Acta Botanica Malacitana, 38, 33-39.

Mittermeier, RA., Tuerner, WR., Larsen, FW., Brooks, JA. \& Gascon, C. (2011). Global biodiversity conservaton: the critical role of hotspots. In: Zachos E, Habel JC eds. Biodiversity hotspots: Distribution and protection of conservation priority areas. Berlin: Springer. 3-22.

Moran, RC., (2008). Diversity, biogepgraphy and floristics. In Ranker TA, Haufler Ch (Eds) Biology and Evolution of Frens and Lycophytes. Cambridge University Press, Cambridge, 367-394.

Pignatti, S., (1982. reprint 1997). Flora d'Italia. Edagricole, Bologna (IT), 3Vols.

PPG1 - Pteridophyte Phylogeny Group, (2016). A community-derived classification for extant lycophytes and ferns. Journal of Systematics and Evolution, 54, 563-603.

Prelli, R., (2001). Les fougères et plantes alliées de France et d'Europe occidentale : Ed. Belin, Paris. 432p.

Pryer, K., Schuettpelz, E., Wolf, P., Schneider, H., Smith, A. \& Cranfill, R. (2004). Phylogeny and evolution of ferns (monilophytes) with a focus on the early leptosporangiate divergences. American Journal of Botany, 91, 1582-1598.

Quézel, P. \& Santa, S. (1962). Nouvelle flore de l'Algérie et des régions désertiques méridionales. Tome I, CNRS, Paris, $636 \mathrm{p}$.

R Core Team, (2013). R: A language and environment for statistical computing. R Foundation for statistical computing, Vienna, Austria. http://www.R-project.org

Raunkiaer, C., (1934). The life forms of plants and statistical plant. Geography. Claredon press, Oxford.

Slimani, AR., Ali Ahmed, M., Hamel, T. \& Coste, C. (2013). Contribution à l'étude de la flore lichénique dans la zénaie de Bougous (forêt de Ramel Toual) au niveau du Parc National d'El Kala Nord Est Algérien. Revue Synthèse des Sciences et de la Technologie, 27, 22 - 29.

Smith, A., Pryer, K., Schuettpelz, E., Korrall, P., Schneider, H. \& Wolf, P. (2006). A classification for extant ferns. Taxon, 55, 705-731.

Stevenson, A., Skinner, J., Hollis, G. \& Smart, M. (1988). The El Kala Park and environs, Algeria: An ecological evaluation. Environmental Conservation, 15 (4), 335348.

Ter Braak, C.J.F. (1995). Ordination: Data analysis in community and landscape ecology. in: R.H.G. Jongman, C.J.F. Ter Braak \& O.F.R. Van Tongeren (eds). Ordination: Data analysis in community and landscape ecology. Cambridge University Press, Cambridge.

UICN. (2017). Liste rouge de I'UICN des espèces menacées. Version 2017-3. <www.iucnredlist.org >

Véla, E. \& Benhouhou, S. (2007). Évaluation d'un nouveau point chaud de biodiversité végétale dans le Bassin méditerranéen (Afrique du Nord). Comptes Rendus Biologies, 330, 589-605.

Vila, J.M., (1980). La chaîne alpine d'Algérie orientale et des confins algéro-tunisienne. Thèse de Doctorat es science Naturelle, Université de Pierre et Marie curie. Paris VI. France. 296 p.

Walter, K. \& Gillett, H. (1998). IUCN red list of threatened plants. Compiled by the World Conservation Monitoring Centre. IUCN - the World Conservation Union. Gland, Switzerland \& Cambridge, UK.

Whigham, DF. (2004). Ecology of woodland herbs in temperate deciduous forests. Ann. Rev. Ecol. Evol. Syst., 35, 583-617.

Yahi, N., Véla, E., Benhouhou, S., de Bélair, G. \& Gharzouli, R. (2012). Identifying Important Plants Areas (Key Biodiversity Areas for Plants) in northern Algeria. Journal of threatened Taxa, 4, 2453-2765. 